\title{
Error Analysis and Calibration for a Novel Pipe Profiling Tool
}

\author{
William Jackson, Gordon Dobie, Charles MacLeod, Graeme West, Carmelo Mineo and Liam McDonald
}

\begin{abstract}
Integrity of industrial pipework is ensured through routine inspection. Internal visual inspection tools are capable of characterising degradation in the form of corrosion, pitting, erosion and cracking. The accuracy of such inspection systems has a direct impact on decisions regarding the remaining lifetime of the asset. By minimising error margins, the asset may be operated with confidence for longer, with less uncertainty. This paper considers a probe system consisting of a laser profiler and camera that produces a textured 3D model of the internals of 2 - 6 inch pipework. The accuracy of the system is defined by the ability to extract laser projections from an image as it travels down the pipe, to accurately reconstruct these projections into 3D and to estimate the probe trajectory as it travels through the pipe. This paper presents an error model of the laser profiler. It then presents a novel calibration routine to reduce the error caused by misalignment and tolerances during fabrication of the system. A key advantage of the proposed calibration technique over alternatives is that we can calibrate for errors without manually adjusting the probe, which enables fabrication of a smaller more robust measurement system. In lab-based trials our calibration technique reduced peak sizing errors from $2.7 \mathrm{~mm}$ to $0.14 \mathrm{~mm}$ in $120 \mathrm{~mm}$ diameter pipes.
\end{abstract}

Index Terms-Visual Inspection, Laser Profiler, Calibration, Pipe Scanning

\section{INTRODUCTION}

$\mathbf{R}$ EMOTE visual inspection (RVI) of pipework within the nuclear industry is a periodic activity carried out to ensure safe operation of the plant. This becomes even more crucial in today's climate as more plants are reaching the end of their expected lifetime and are being extended to operate up to and possibly beyond an additional twenty years [1]. Currently such commercial inspection is typically carried out by an operator monitoring a live or recorded video feed of the pipe interior delivered by rigid cable, tractor, or endoscope style devices. There are issues associated with this traditional method including lack of orientation awareness within the pipe due to minimal visual references within the pipe. Errors related to location within the pipe also tend to increase with distance travelled, this is due to the common mode of measurement where insertion length is directly related to encoder turns which is susceptible to slippage and thus mismeasurement.

Multiple passes of the pipe may also be required at different viewing angles as opposed to the single pass required by the system presented in the paper due to the restricted field of view of typical video feed systems. Although stereo based systems are available the most commonly used RVIs cannot directly

The authors are with the Department of Electronic and Electrical Engineering, University of Strathclyde, Glasgow G1 1XQ, U.K. (e-mail: william.jackson@strath.ac.uk).

Manuscript received August 02, 2019; revised November 14, 2019; accepted December 11, 2019 size defects which leads to subjective errors arising from differences of interpretation. Research has been carried out in reconstructing the geometry of the pipework using monocular vision which addresses these issues and provides quantitative RVI. This research can be divided into two sub-categories, the first using structure from motion (SFM) to determine the pipe structure [2]-[4]. The second category which will be the main focus utilises a projected laser ring to infer the geometry of the pipe [5]-[11].

The RVI system used in this paper was a laser profiler and omni-directional camera unit capable of sizing defects to sub $\mathrm{mm}$ levels and providing highly accurate locational information by utilising a combination of visual odometry [12], encoders and an inertial measurement unit. The combination of the geometric information provided by the laser profiler and omni-directional camera images allows for a textured mesh to be created from a combination of both sets of data, generating a $3 \mathrm{D}$ reconstruction of the pipe interior.

Calibration of the laser alignment with respect to the camera is crucial as it directly impacts the sizing ability of the probe. In the majority of cases from the literature, alignment is assumed to be ideal and no calibration procedure is defined. There are however some examples, Zhu et al. [10] detail a calibration technique for a similar system to the authors'. It is however not suitable due to requiring the laser ring to be projected in front of the camera onto a target as opposed to that used in this paper which is orthogonal to the camera. Another method is shown by $\mathrm{He}$ et al. [11], a benefit of this calibration method is that it corrects for distortion caused by the supporting glass tube linking the camera to the laser projector.

A method of calibration which could be implemented is that demonstrated by Buschinelli et al. [9], in the procedure presented the profiler is mounted upon stages inside a highly accurate calibration artefact. The artefact consists of a series of known step sizes in a cone like geometry, a polynomial is calculated for each angle of the imaged laser ring in images taken at for step of the calibration artefact and interpolated for the full sizing range of camera. This provides a function for each pixel in the image mapping to a certain radius.

This paper introduces a new technique for alignment of the camera and laser module allowing for correction of angular misalignment as well as translation with manual adjustment of the laser profiler. The procedure allows for correction of angular error without manual adjustment, making it suitable for production ready systems.

An advantage of the proposed technique when compared to [9] is that the calibration is performed with a single capture vs approximately 30 with the use of an arguably simpler set- 
up and calibration object than that used by Buschinelli et al which is shown in Fig. 1a. When compared to [11] there is no need for an additional camera and complexities this adds to the calibration the experimental set up is shown in Fig. 1b. Furthermore, the calibration method presented by $\mathrm{He}$ et al. required 29 images to be taken of a chequerboard in various positions increasing the time and effort required whereas the calibration method which will be presented in this paper only requires a single image with no additional camera. He et al. report an error of $0.23 \mathrm{~mm}$ when measuring a $288 \mathrm{~mm}$ pipe, Buschinelli et al. report an error of $0.2 \mathrm{~mm}$ on $100 \mathrm{~mm}$ diameter pipe sections. These values can be compared to the error of the authors system of $0.14 \mathrm{~mm}$ in a $120 \mathrm{~mm}$ diameter pipe.

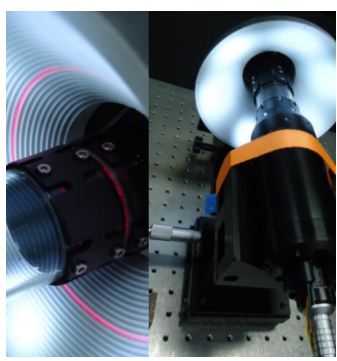

(a)

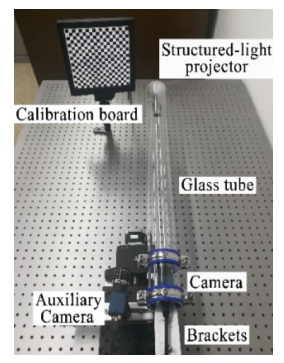

(b)
Fig. 1. Alternative calibration set-ups: (a) shows that used by Buschinelli et al. [9] and (b) shows that of He et al. [11]

\section{BACKGROUND}

The probe discussed in this paper consists of a $2 \mathrm{MP}$ machine vision camera with a fish-eye lens and laser profiler. The fish-eye lens provides $180^{\circ}$ field of view, thus in a single image a $360^{\circ}$ section of the pipe is captured, for $75 \mathrm{~mm}$ radius pipe around $100 \mathrm{~mm}$ deep into the pipe is visible from a single image. This leads to quicker inspections, as traditional video probe footage may need to be repeated with the probe at multiple orientations within the pipe to ensure full coverage.

The current hardware prototype, deployable in 2-6 inch diameter pipe up to lengths of $50 \mathrm{~m}$ is shown in Fig. 2. A model detailing the geometry of the probe is shown in Fig. 3.

During inspection the probe alternates between an LED image and a laser image. The illuminated LED image captures the texture and features, which are used for tracking the position of the probe within the pipework. The laser images provide direct geometric information, example images captured by the probe are shown in Fig. 4.

The laser image is used to profile the interior of the pipe under inspection, the probe projects a laser line onto the circumference of the inner pipe wall and images this while being driven through the pipe. These images are then used to determine the geometry of the pipe and thus detect surface defects such as cracks and pitting as well as providing information on geometric defects such as large areas of corrosion.

With regards to the aforementioned geometry of laser projector and camera as shown in Fig. 3, $r$ is the pipe radius, $\theta$ describes the angle between the central axis and the imaged ray, $\mathbf{P}_{\mathbf{w}}$ is the point in space where the laser intersects the pipe

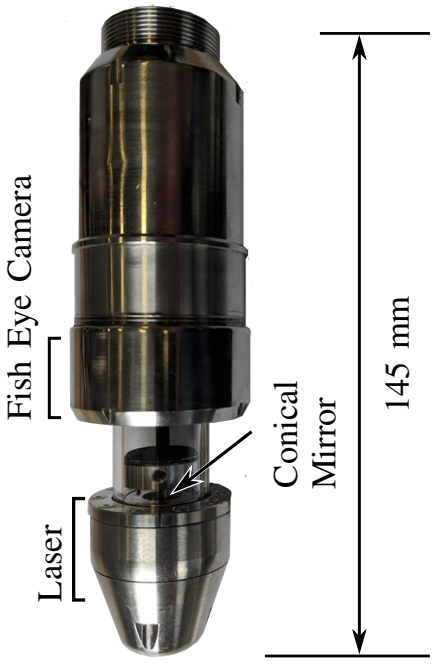

Fig. 2. Camera and laser profiler with subsystems highlighted

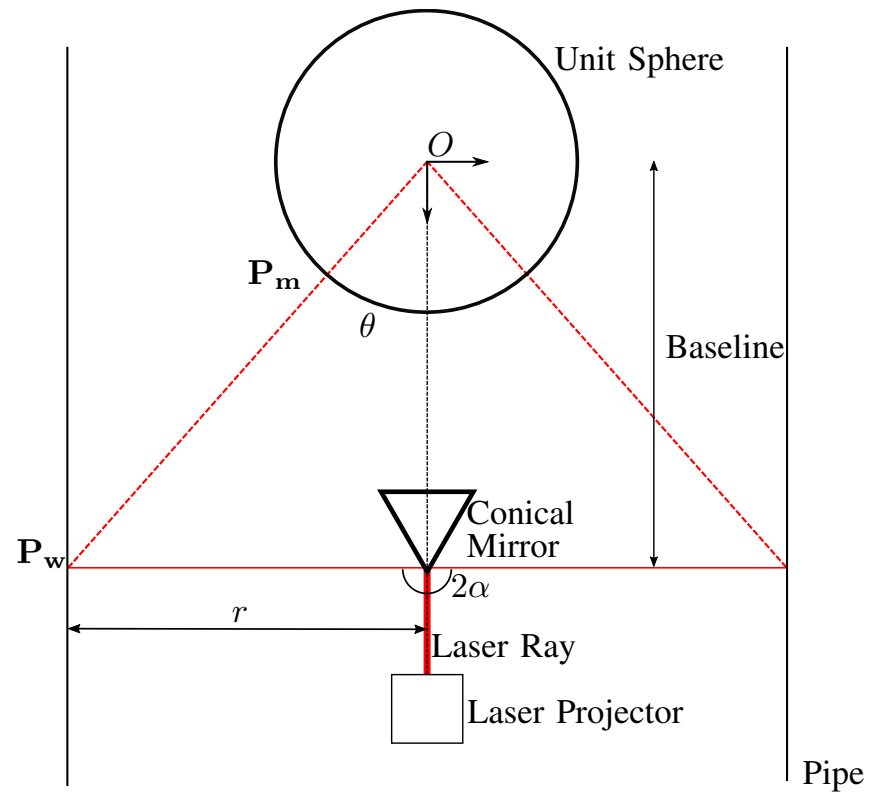

Fig. 3. Camera and laser profiler geometry

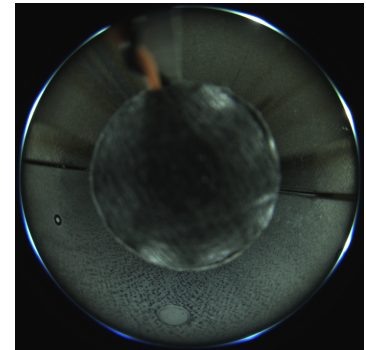

(a)

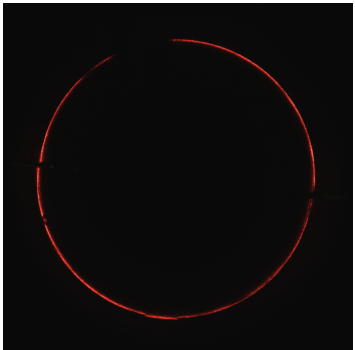

(b)
Fig. 4. Example images taken with the probe in a split pipe sample with a flat bottom hole: (a) shows the images under LED illumination and (b) shows the projected laser line 
wall, $\mathbf{P}_{\mathbf{m}}$ is the point on the unit sphere (a representation of vectors entering the camera centre at one-unit distance) where this point is imaged and $\alpha$ which is the opening angle of the laser reflected by the conical mirror. The baseline is the distance between the origin $O$ and the point of reflection. It can be observed from the model that once the values of the baseline and opening angle $(\alpha)$ are known for a given point on the unit sphere $\left(\mathbf{P}_{\mathbf{m}}\right)$, the corresponding point $\mathbf{P}_{\mathbf{w}}$ can be calculated through triangulation. To calculate $\mathbf{P}_{\mathbf{m}}$ from a given pixel on the image the camera model developed by Scaramuzza et al. [13]-[15] is used. Scaramuzza introduced a central projection model which converts the pixel point on the image into a bearing vector giving the direction of the imaged ray entering the central point of the camera. These camera model parameters are obtained with an improved calibration technique by Urban et al. [16]. The accuracy of the reprojected laser point is determined by several different sources which are summarised as follows:

1) Systematic Error

a) Misalignment - manufacturing tolerances, optical axis and laser misalignment

b) Opening angle $(\alpha)$

c) Baseline length

d) Intrinsic camera properties

2) Sampling Error

a) Variable laser line width

b) Photo-sensor noise

The focus here will be on the alignment error and corresponding variation of $\alpha$ around the $360^{\circ}$ the probe.

\section{AlignMENT ERROR}

Systematic errors occur due to differences between the model given in Fig. 3 and the manufactured probe. These differences have a direct impact on the accuracy of the measured diametric information of the sample under inspection. It is assumed in current the model that the laser plane is parallel to the image plane i.e. constant $\alpha$ of $90^{\circ}$ revolved around the model. Due to manufacturing tolerances, angular misalignment may be introduced into the system resulting in sizing errors. To illustrate this a diagram detailing how these errors arise due to misalignment is shown in Fig. 5. In this case the probe is assumed to be centred within the pipe and the only source of error is due to angular misalignment of the conical mirror.

The diagram shows the expected laser line given as the vertex between $P_{c}$ and $P_{c}^{\prime}$ and the actual laser line $\left(P-P^{\prime}\right)$ resulting from the angular error $(\phi)$ of the conical mirror. The assumed opening angle is $90^{\circ}$, shown in the diagram as $\angle O C P_{c}$. The angles theta $(\theta)$ and thet $a^{\prime}\left(\theta^{\prime}\right)$ are the angles from the respective imaged points $P$ and $P^{\prime}$ to the camera centre. In the case where the opening angle is assumed to be $90^{\circ}$ the point through triangulation is that where the ray drawn from $O$ at angles $\theta$ and $\theta^{\prime}$ intersect the line $P c-P_{c}^{\prime}$. These points of intersection are labelled as $E_{l}$ and $E_{r}$ as the positive and mirrored negative $\phi$. The resulting diameter is shown as the dimension $E$ it is noted that there is a considerable translation error introduced as well as a sizing error shown

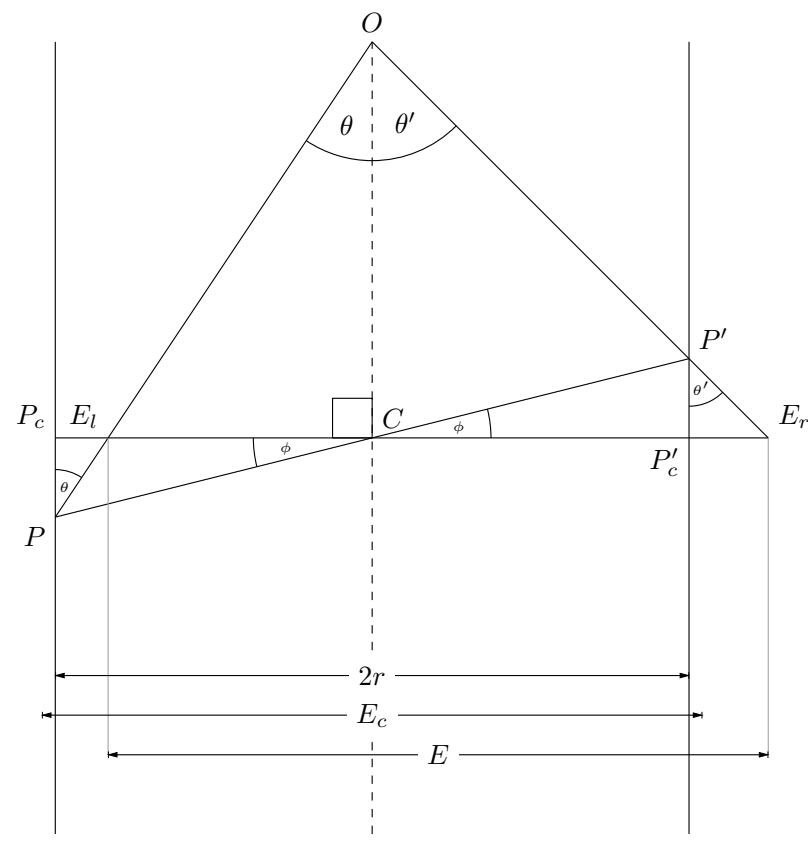

Fig. 5. Illustration detailing error in sizing of pipe radius $(r)$ due to misalignment angle $\phi$

more clearly in $E_{c}$ where the dimension $E$ has been placed on the central axis.

\section{Alignment Procedure}

As described previously, alignment of the laser and camera are of critical importance for accurate laser measurements. When the laser and image plane are misaligned, the radial distance observed by the camera is not its true value. An overview of the alignment procedure to ensure alignment is detailed below:

1) Align camera to calibration block

2) Align laser ring within calibration block

3) Align laser centre within calibration block

To align the laser profiler and camera they are both referenced to the same calibration object shown in Fig. 6.

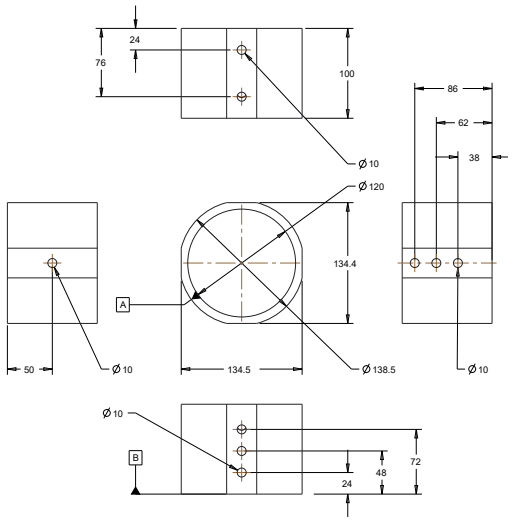

Fig. 6. Technical drawing of calibration block used for alignment of camera and laser profiler 
The calibration object consists of an accurately machined block with nine flat bottomed holes each with a point marker in the centre. These centre targets are to be extracted and used to align the camera relative to the block. Features within the block are recessed to allow accurate scanning of their relative centre points with a Faro Arm portable co-ordinate measurement machine. A ball-end probe was traced around each of the inside diameters of the flat bottomed holes gathering a set of points representing the location of the features in 3D. A circle was then fitted to these points, the centre point of these circles would then serve as the ground truth for the 3D position of each feature point in space. To allow accurate positioning of the camera relative to the block a series of three stages (two translation and one tip-tilt) were used to give the camera the four degrees of freedom required for alignment within the calibration block. Roll around the camera axis and travel along this axis are not required. A further tip-tilt stage is integrated with the laser projector of the probe to allow adjustment of the laser.

This tip-tilt stage is mounted on a slotted fixture to allow horizontal translation and shims were used to allow for vertical displacement, providing the same degrees of freedom as the camera unit allowing the laser projector to be aligned with the calibration block. The probe and stages are detailed in Fig. 7.

\section{Top View}
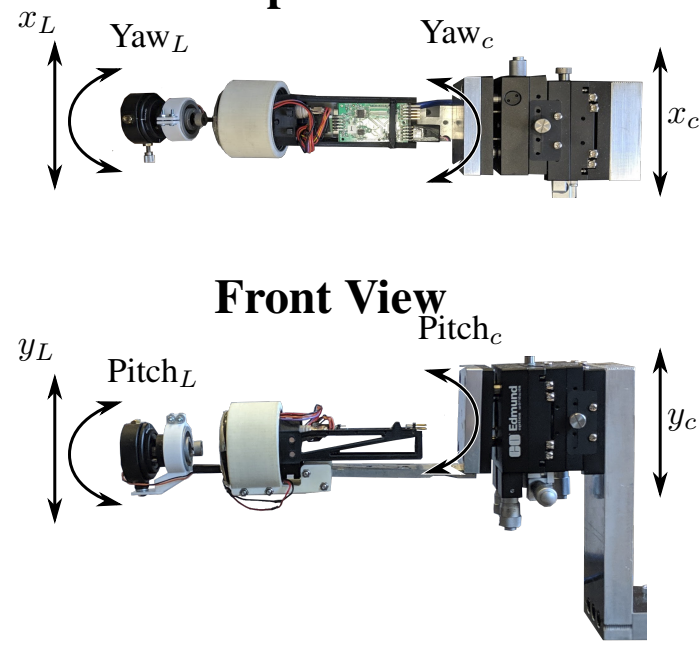

Fig. 7. Fisheye camera and laser profiler mounted to adjustment stages for alignment within calibration block with each stage's degree of freedom labelled

The method used to position the camera in 3D space within the calibration block is the Perspective N-Points $(\mathrm{PnP})$ algorithm, specifically developed by Urban et al. [17]. As the points of the test block are captured arbitrarily in space, they need to be transformed into a relative co-ordinate frame. This co-ordinate frame is based on the centre and axis vector of a fitted cylinder through the gathered points. To calculate the parameters of the cylinder, one must be fitted to the nine points this is achieved through use of the PROTO toolbox [18]. The main parameter of interest is the axis of the cylinder which fits these points as it will be used as the $Z$-Axis in the new coordinate system. This along with a chosen point on the cylinder (in this case the point which is the lone feature on one side of the test block detailed Fig. 6) is the basis of a co-ordinate system which will be used for positioning the camera. The origin is to be described as the point of intersection between a perpendicular line from the selected point and the axis of the cylinder. The vector describing this line is to be the $\mathrm{X}$-axis and is described by the following equation (1):

$$
\vec{x}=(\overrightarrow{P A})-((\overrightarrow{P A}) \vec{C}) \vec{C}
$$

Where $\overrightarrow{P A}$ is the vector between point $P_{x y z}$ as the selected point on the pipe, $A_{x y z}$ is the origin of the fitted cylinder and $\vec{C}$ is the vector describing the cylinder axis. The point of intersection and thereby the origin of the system is given by (2).

$$
O_{x y z}=\vec{x}+P_{x y z}
$$

The $Y$-Axis is the cross product of the $Z$ and $X$-Axis vectors. A co-ordinate system has now been described by the cylinder, however it would be more desirable for the origin to be at $0,0,0$ and the $Z$-Axis to be equal to $[0,0,1]$. To achieve this a basis transformation is performed, a homogeneous translation matrix based upon the axis of the system as shown in (3) is created. This is then applied to the captured points denoted as $D$ in (4).

$$
\begin{gathered}
T=\left[\begin{array}{cccc}
x_{0} & y_{0} & z_{0} & O_{0} \\
x_{1} & y_{1} & z_{1} & O_{1} \\
x_{2} & y_{2} & z_{2} & O_{2} \\
0 & 0 & 0 & 1
\end{array}\right] \\
\text { Points }=T^{-1}\left[\begin{array}{ccccc}
D_{00} & D_{10} & D_{20} & \ldots & D_{n 0} \\
D_{01} & D_{11} & D_{21} & \ldots & D_{n 1} \\
D_{02} & D_{12} & D_{22} & \ldots & D_{n 2} \\
1 & 1 & 1 & \ldots & 1
\end{array}\right]
\end{gathered}
$$

After this operation the points and fitted cylinder are observed as shown in Fig. 8.
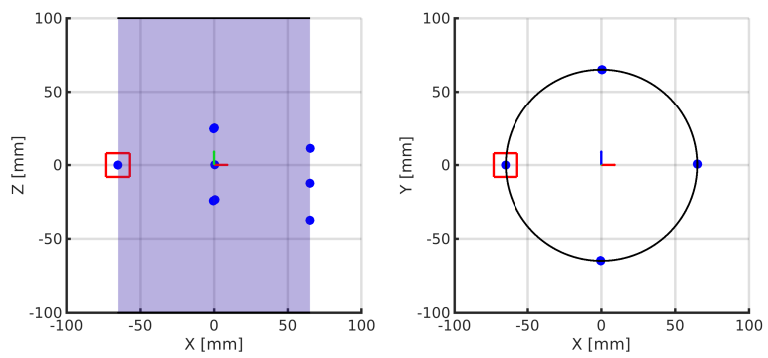

Fig. 8. Feature points with respect to the co-ordinate frame used for determining the relative position of the probe within the calibration block, the selected point for positioning the co-ordinate frame is highlighted

These points and relative co-ordinate frame may now be used as a reference to position the probe co-linearly with the calibration block. To extract the centre point of the features for input into the PnP algorithm, a small black dot on a white background is located centrally within each recession 
of calibration block. An example image showing the features taken within the calibration block is shown in Fig. 9.

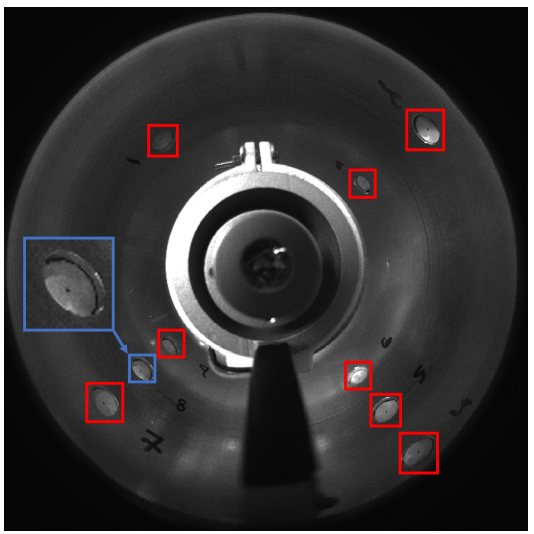

Fig. 9. Image taken by probe within calibration block, showing the nine features (highlighted) used to centre the probe within the block using the PnP algorithm

To extract the centre point, first a region of interest is drawn around each feature. For each region of interest, the corner detector developed by Shi and Tomasi [19] is implemented to locate the centre.

An example input image Fig. 10a and corner response Fig. 10b are shown, a threshold of $70 \%$ is applied to the image and the centre of the central region is deemed to be the location of the feature point as marked by a cross as in $10 \mathrm{~d}$. This threshold value was obtained experimentally.

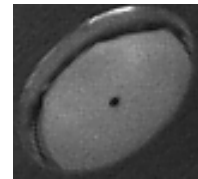

(a)

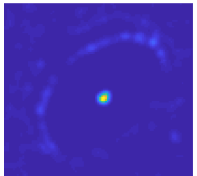

(b)

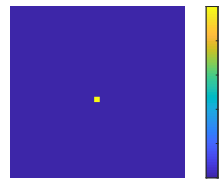

(c)

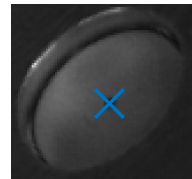

(d)
Fig. 10. Example extraction of the region of interest around a localising feature of the calibration block, (a) shows the input image, (b) shows the corner response of the image, (c) shows the response after applying the threshold and (d) shows the location of extracted centre

The process for aligning the camera is shown in Fig. 11, the camera is deemed to be aligned when the observed $X \& Y$ values were in oscillation around 0 . In translation the camera was positioned within $0.1 \mathrm{~mm}$ with a standard deviation of $0.03 \mathrm{~mm}$ in $X$ and $Y$ over 100 repetitions of acquisition and processing.

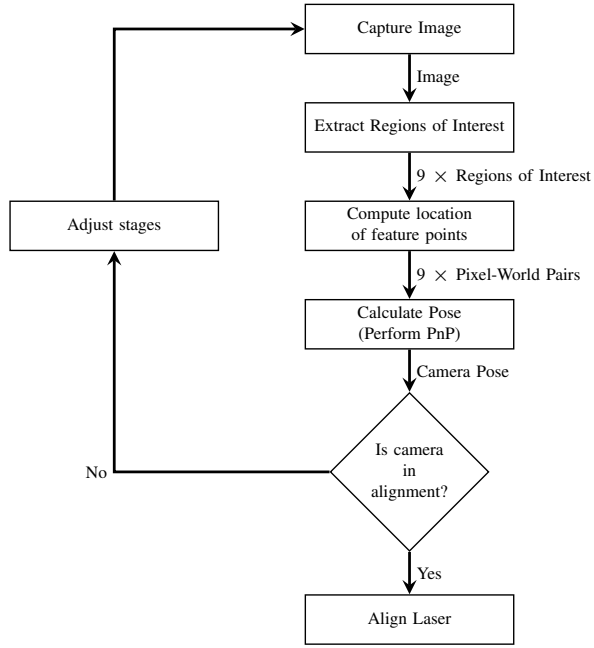

Fig. 11. Procedure for aligning camera within calibration pipe

\section{LASER Alignment}

With the camera and pipe in alignment the laser may now be aligned to the common calibration block. The first step in this process is extracting the laser line in the image, this is achieved through the following steps:

1) Define a circle whose diameter is that of the image width

2) Plot the intensity from the image centre to each point on the circle

3) Extract the peak value along this line

Lines of extraction between the two points are shown in Fig. 12 and the intensity along an example line is plotted in Fig. 13. The laser alignment is completed in two steps, the

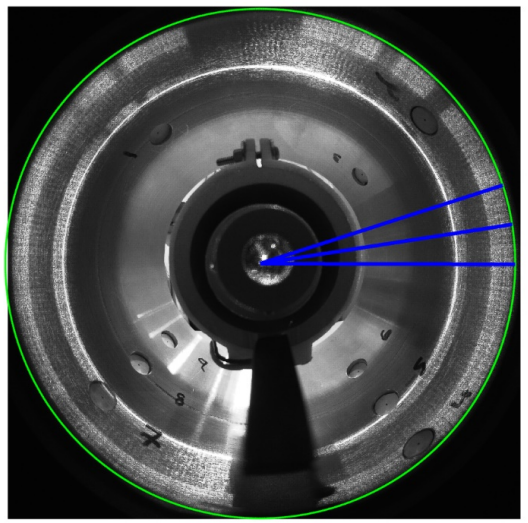

Fig. 12. Image detailing extraction of the laser line, the green circle illustrates the extents of extraction, the blue lines show example extractions of intensity going around the full circumference

first is to align the laser plane. Alignment of the laser plane is achieved by adjusting the angular stages of the laser module as shown in Fig. 7. For a measure of angular alignment to use during the process which was relatable to the adjustment of the stages, a circle was fitted to the extracted laser ring. The centre of this circle could be used as a reference for alignment with the error being the distance from the image centre. The yaw adjustment allowed adjustment in $X$ and the pitch adjustment allowed for adjustment in $Y$. The stages were adjusted until 


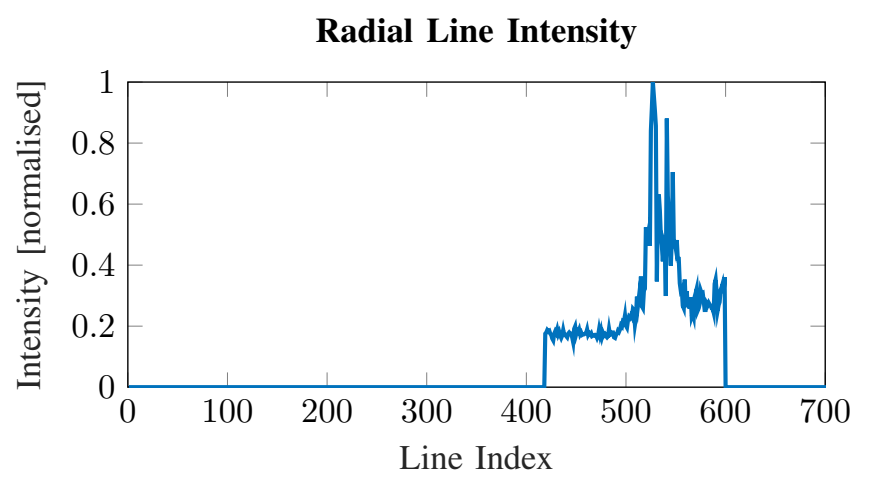

Fig. 13. Extracted intensity line (threshold on index applied)

the fitted circle had a centre equal to the camera centre. When this occurs the laser plane is orthogonal to the pipe axis and parallel to the camera plane. This can be further confirmed by calculating the bearing vector at each point of the circle and ensuring they are equal. In the example aligned image shown in Fig. 14, the extracted circle shown in red has an error from camera centre of 0.44 pixels in $X$ and -0.20 pixels in $Y$. This

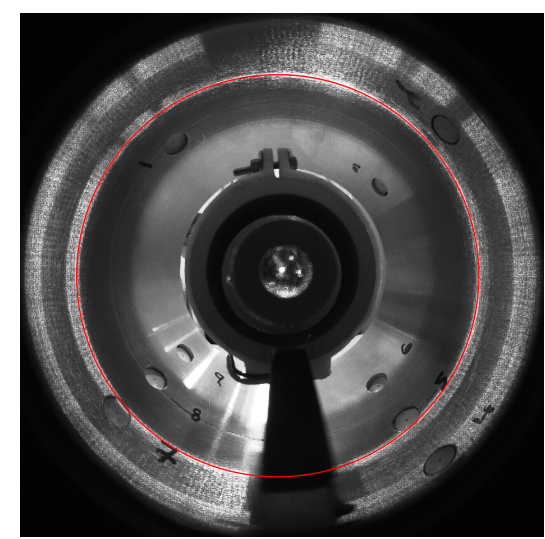

Fig. 14. Aligned laser, circle fit of laser shown in red

slight error can be seen in the aforementioned bearing vector calculation. Across 2000 points of the laser circle the mean angle was $69.9^{\circ}$ with a maximum deviation of $0.09^{\circ}$. This can be compared to a deviation of four degrees in both axis of adjustment in the laser stage, the resulting circle centre was located at 19.1 in $X$ and 24.3 in $Y$. The mean bearing vector value was $70.4^{\circ}$, the maximum deviation was noted as $4.34^{\circ}$. The bearing vector calculated at each extraction point around the laser ring is plotted in Fig. 15. The laser plane can now be assumed to be aligned with an opening angle of $90^{\circ}$ at all points around the image.

In this configuration, the laser's angular alignment is correct. However, there still exists an error in translation. As this body of work is focusing on the error due to angular misalignment this translation error must be corrected so as not to have an impact on the results. To position the laser correctly an external feature is used, this is due to the fact once aligned the source could be placed anywhere within the calibration block and produce the same image due to the planar nature of the projection. The outline of the housing of the conical mirror

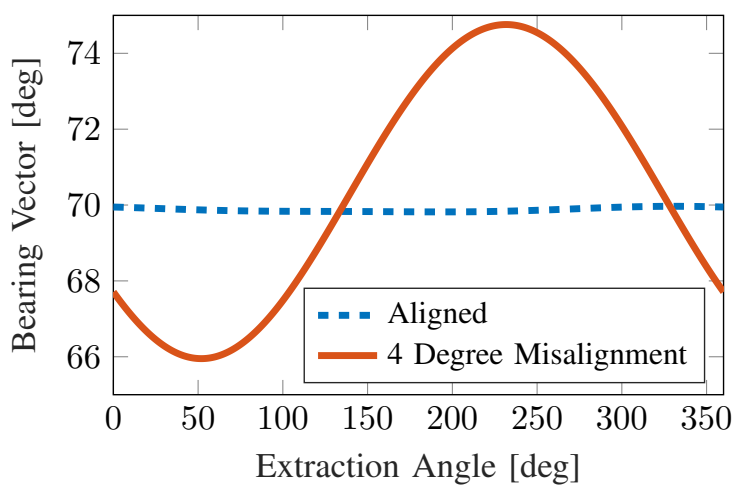

Fig. 15. Calculated bearing vector at each point in laser circle for aligned and misaligned conical mirror

is visible from the camera's perspective, this gives an accurate reference to position the conical mirror in translation. As there is a large contrast difference between this area and the outer housing it can be extracted using a circular Hough transform [20] localised within the region. An example image showing the extracted circle highlighted in blue is shown in Fig. 16. The centre of this extracted circle is compared to the camera centre as performed previously with the angular misalignment. Translation according to Fig. 7 in $Y$ was achieved using shims beneath the module and in $X$ by the virtue of the module being mounted on a slotted plate. Centring of the extracted circle was repeatable to within a pixel of the camera centre. The variation in extraction due to lighting and other factors caused a deviation of \pm 3 pixels over multiple images. Once this procedure is complete the laser is known to be aligned in both orientation and translation with reference to the camera central axis, which itself is aligned to the calibration block.

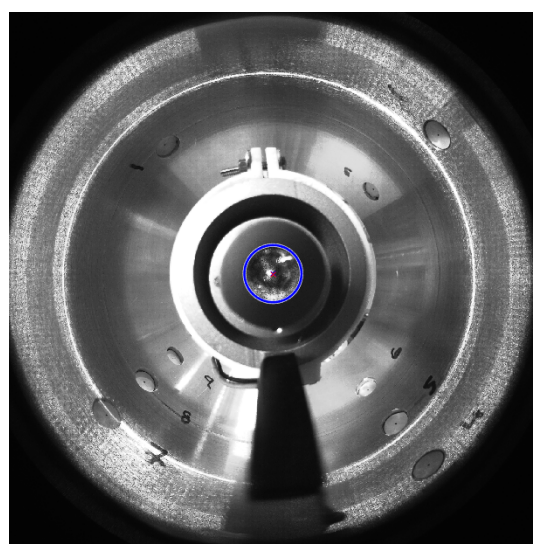

Fig. 16. Centring of conical mirror using circular Hough transform on the mirror outline

\section{LASER MisalignMENT}

With the laser in alignment it is possible to study the angular error in a controlled manner utilising the angular stages housing the laser module. From the aligned position the stage was adjusted to a maximum of $5.5^{\circ}$ in each axis in steps of $0.35^{\circ}$. At each position a laser image was captured, a circle was then fitted to the data to remove any noise in 
the extraction. Each point of the circle was projected into 3D space with an assumption that the opening angle was $90^{\circ}$. Two error values are then calculated, the radius error i.e. the error of the projected circle vs the calibration block and the positional error given as the distance from the origin to the centre of the projected circle. The radius error is shown in Fig. 17 with a $\mathrm{max} / \mathrm{min}$ error of: $1.5 / 0.05 \mathrm{~mm}$. The positional error in Fig. 18 has a max/min error of: $15.8 / 0.12 \mathrm{~mm}$. These results reflect that the error has a much greater impact on the position as opposed to the radius as detailed in Fig. 5. The errors of the system are now deemed to be well understood and it would be advantageous to correct for them without manual adjustment, a method for this will be introduced in the following section.

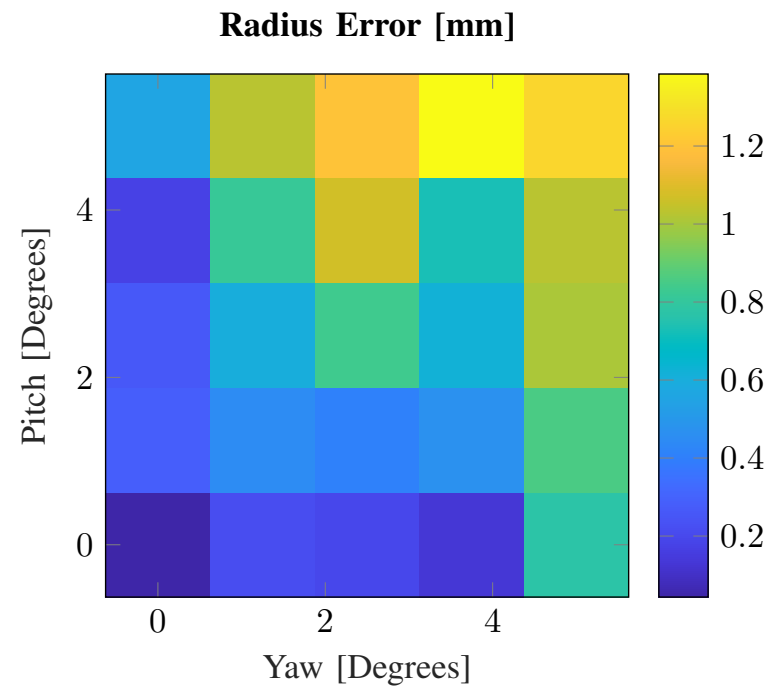

Fig. 17. Radius error across $0-5^{\circ}$ of angular misalignment

\section{Positional Error [mm]}

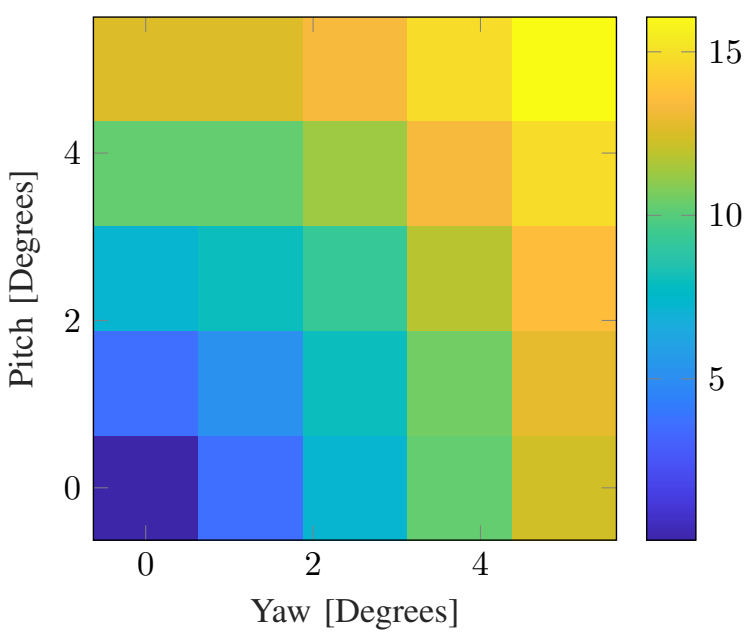

Fig. 18. Positional error across $0-5^{\circ}$ of angular misalignment

\section{LASER CORRECTION}

Prior to the completion of this work the previous method used to calibrate the laser profiler was to estimate the opening angle and baseline using a series of known radii calibration samples. A minimising function was utilised to calculate the opening angle and baseline, these values were assumed to be equal around the entirety of the image leading to the errors shown previously where misalignment was present. With the profiler co-linear with the calibration block a more comprehensive calibration can be performed, correcting for angular misalignment at discreet points through the entire $360^{\circ}$ of the profiler. When centred the origin to each point of extraction on the calibration block is at a known distance and the conical mirror can be assumed to be machined highly accurately to a $45^{\circ}$ angle. Using this information, a two-step calibration procedure can be performed. This is summarised in the following sequence:

1) Extract Laser Ring

2) Calculate bearing vector for opposite points on ring and average (opening angle known to be 90 degrees)

3) Derive baseline from these values

4) Calculate unique opening angles with baseline

The first step in this correction process as with previous examples is to capture an image of the laser ring and fit a circle to the data. Following this the baseline can be calculated for two opposing points on the laser ring for example $0^{\circ} \&$ $180^{\circ}$. Between these two points the opening angle of the laser can be assumed to be a combined value of $180^{\circ}$ due to the known geometry of the conical mirror. For the two extracted points the bearing vector $\left(\mathbf{P}_{\mathbf{m}}\right)$ was acquired using the camera model, $\theta$ as denoted in Fig. 3 can then be calculated (5) where $\mathbf{C}$ denotes the camera axis $[0,0,-1]$.

$$
\theta=\arccos \left(\frac{\mathbf{P}_{\mathbf{m}} \cdot \mathbf{C}}{\left\|\mathbf{P}_{\mathbf{m}}\right\|\|\mathbf{C}\|}\right)
$$

By calculating theta for both extraction points and taking the average a synthetic point is created with an effective opening angle of $90^{\circ}$ this can then be used to calculate the baseline length $(B)$ for these two points using the known radius of the calibration block $(R)$.

$$
B=\frac{R}{\tan \theta_{\text {avg }}}
$$

At this point for all extraction points of the laser ring a baseline is known and unique opening angles $(\alpha)$ can be calculated (in the following theta is calculated for each point independently).

$$
\alpha=\pi-\theta-\arcsin \left(\frac{B \sin \theta}{R}\right)
$$

There now exists $n$ baseline $(B)$ values and $n \alpha$ values for each angle of extraction around the laser image. Calculating a world point $\left(\mathbf{P}_{\mathbf{w}}\right)$ from a given mirror point $\left(\mathbf{P}_{\mathbf{m}}\right)$ and its associated $\theta$ value at extraction index $i$ is given by (8).

$$
\mathbf{P}_{\mathbf{w}}=\mathbf{P}_{\mathbf{m}} \frac{B[i] \sin (\alpha[i])}{\sin (\pi-\theta-\alpha[i])}
$$


The effect of this calibration method is illustrated in Fig. 19 where the laser profiler was set to have a four degrees angular error, the left images denoted Global Angle shows the large positional error and the laser being projected parallel to the calibration block ends. On the right side of the image labelled as Multi Angle the positional error has been corrected and the laser is projected at the true angle.

As the profiler is calibrated against the current calibration block to be able to compare its performance with the previous method of calibration it was tested across a series of pipes with radii: $37.41 \mathrm{~mm}, 50.90 \mathrm{~mm}, 57.20 \mathrm{~mm}$, and $63.43 \mathrm{~mm}$. Each pipe and angle combination consisted of 10 sample images with the probe repositioned within the pipe between each frame. Due to not knowing the position of the profiler within the pipes only the radius error can be measured. The resulting errors for both the Multi Angle or calibrated and Global Angle are detailed in Fig. 20.

Average error with Multi angle (corrected): $-0.29 \mathrm{~mm}$ Average error with global angle (uncorrected): $2.19 \mathrm{~mm}$
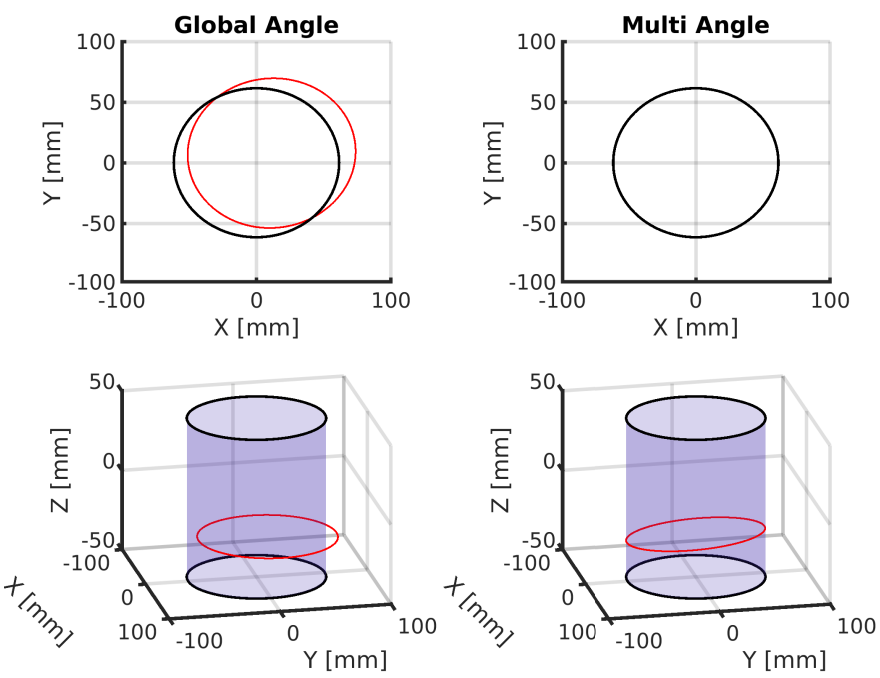

Fig. 19. Calibrated and un-calibrated laser extraction at $4^{\circ}$ misalignment within calibration block
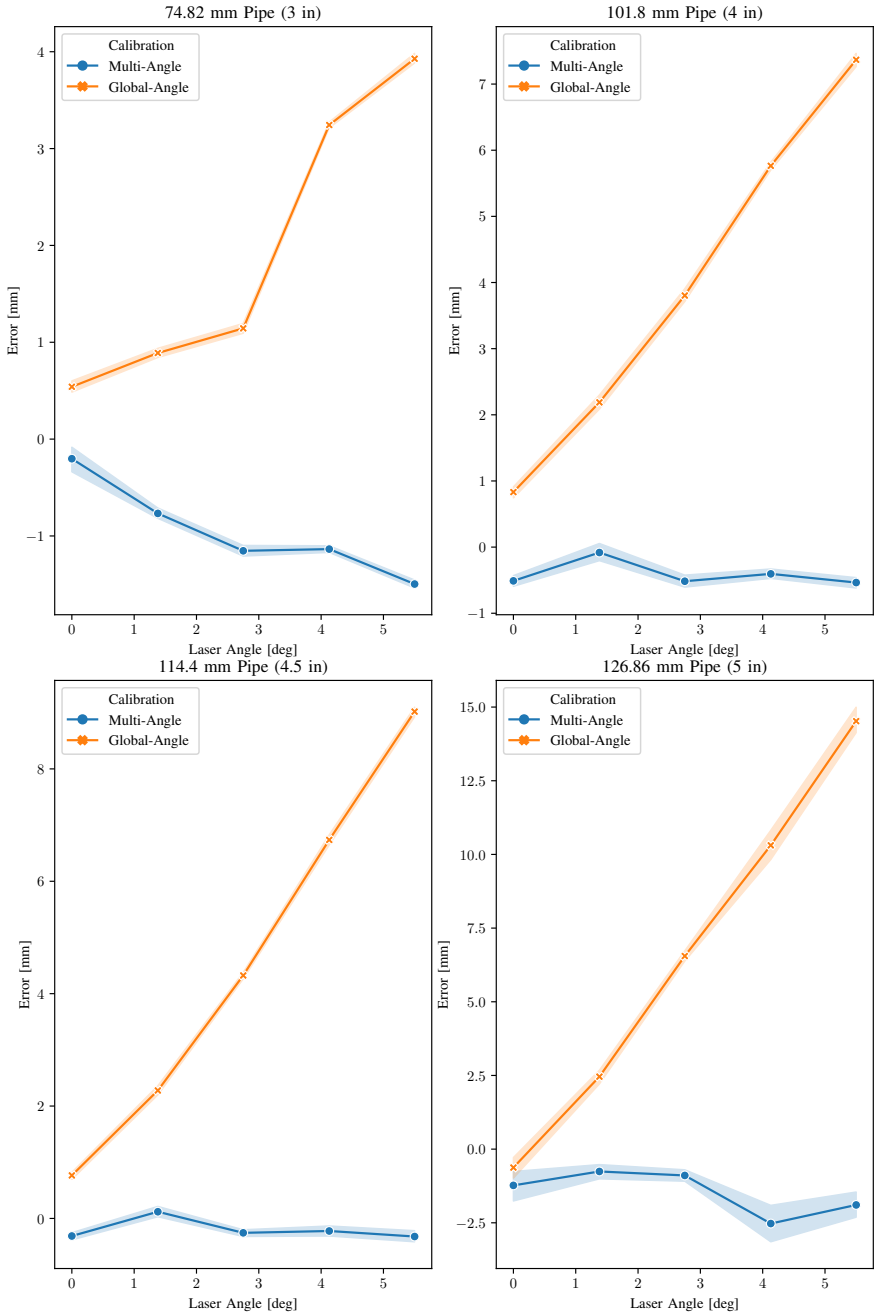

Fig. 20. Grouped error plots of pipe diameter sizing for increasing misalignment angles of laser profiler

\section{COMPARISON to Ground Truth Model}

To determine the suitability of the calibration method and laser scanning for practical applications a controlled scan was performed over a split pipe test sample with flat bottom hole defects as shown in Fig. 21. The reference scan which the results will be compared to was generated by a GOM ATOS Triple Scan system, capable of providing accuracy of $20 \mu \mathrm{m}$ [21]. The probe shown in Fig. 7 was mounted onto a robotic end effector and driven centrally through the split pipe recording alternating LED and laser images to repeatably simulate manual deployment.

The LED images were processed to provide the positioning of the laser scanner within the pipe using feature-based matching [22], it has been previously shown that this technique is capable of providing positional feedback accurately to $0.01 \mathrm{~mm}$ per $\mathrm{mm}$ travelled [12]. Each laser image from the recording had the laser line projected into 3D space using both the Global and Multi Angle calibration methods, this process produced a series of rings which were then translated in space using the corresponding positional information obtained from the LED image set. 
Comparison with the GOM model was made possible by meshing the extracted points utilising the algorithm detailed in [23]. This meshing algorithm remains true to the original data as each point directly becomes a vertex on the mesh. A drawback of meshing the data is that error is introduced around the edges of the holes due to the steps between consecutive rings creating a slope into the hole as opposed to a discrete change. Resulting deviation maps are shown in Fig. 22, these deviation maps are created by GOM Inspect Software which aligns the ground truth mesh with those generated by the laser probe.

From the deviation maps it clear that in conditions of misalignment the correct aspect ratio of the flat bottom holes is lost with an over-sizing of diameter in the horizontal dimension. The accuracy of the axial dimension is given by the visual odemetry in both cases of aligned and misaligned. In depth sizing the largest errors were observed on the $10 \mathrm{x}$ $2 \mathrm{~mm}$ flat bottom hole this data is presented in Table I. In the table All refers to the mean and standard deviation error of the entirety of the sample, the error for the flat bottom holes consists of the mean error and standard deviation for a patch of selected points centred in the hole. Diametric error was calculated by observing the error along a plane that intersected the widest section of the hole with the error points taken at the upper edge of the flat bottom hole. It is observed that under maximum misalignment the calibration approach proposed reduces diameter errors of a $10 \mathrm{~mm}$ hole from $2.50 \mathrm{~mm}$ to $0.27 \mathrm{~mm}$ and depth measurements of a $2 \mathrm{~mm}$ deep hole from $-0.96 \mathrm{~mm}$ to $-0.21 \mathrm{~mm}$.

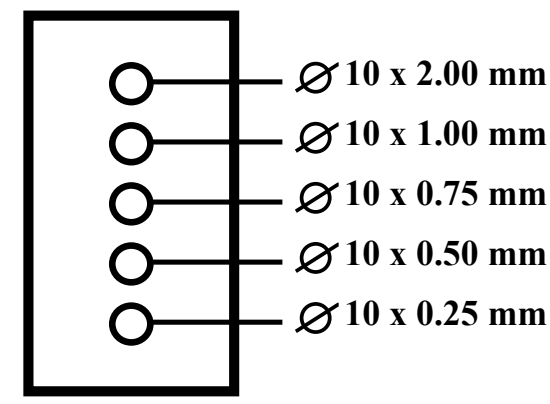

Fig. 21. Technical drawing of split pipe sample

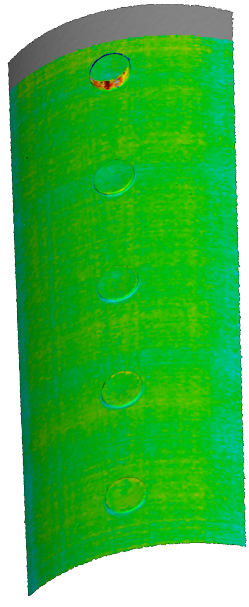

(a)

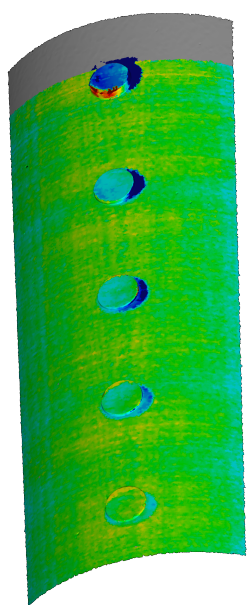

(c)

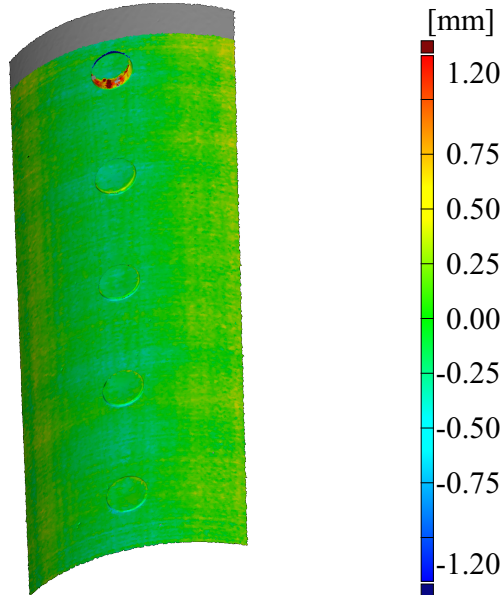

(b)

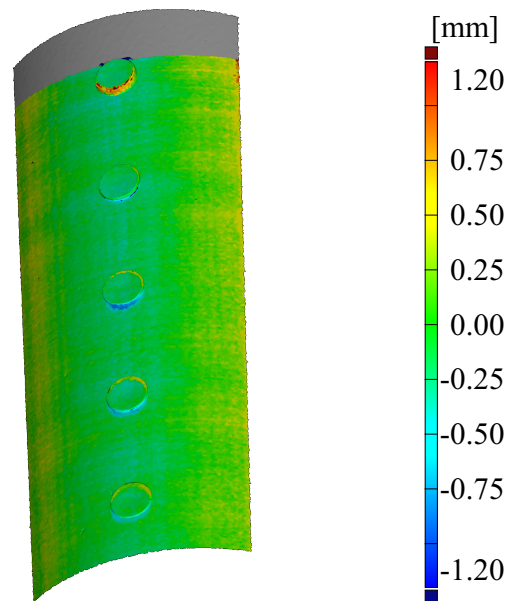

(d)
Fig. 22. Deviation maps showing error between generated mesh and ground truth scan (a) shows the Global Angle calibration error at 0 degrees misalignment and (b) shows the Multi Angle, (c) shows the Global Angle calibration error at 5.5 degrees and (d) shows the equivalent using Multi Angle calibration 
TABLE I

SPLIT PIPE ERROR

\begin{tabular}{|c|c|c|c|c|c|c|c|c|c|c|}
\hline \multirow[b]{3}{*}{ Misalignment [deg] } & \multicolumn{5}{|c|}{ Global Angle } & \multicolumn{5}{|c|}{ Multi Angle } \\
\hline & \multicolumn{2}{|c|}{ All } & \multicolumn{3}{|c|}{$10 \times 2 \mathrm{~mm}$ Hole } & \multicolumn{2}{|c|}{ All } & \multicolumn{3}{|c|}{$10 \times 2 \mathrm{~mm}$ Hole } \\
\hline & Mean $[\mathrm{mm}]$ & SD. $[\mathrm{mm}]$ & Mean [mm] & SD. $[\mathrm{mm}]$ & Diam. Err. $[\mathrm{mm}]$ & Mean [mm] & SD. $[\mathrm{mm}]$ & Mean $[\mathrm{mm}]$ & SD. $[\mathrm{mm}]$ & Diam. Err. [mm] \\
\hline 0 & -0.02 & 0.19 & -0.11 & 0.12 & 0.20 & 0.02 & 0.22 & -0.16 & 0.08 & 0.16 \\
\hline 1.4 & -0.04 & 0.26 & -0.32 & 0.09 & 0.77 & 0.01 & 0.22 & -0.19 & 0.07 & 0.22 \\
\hline 2.8 & -0.04 & 0.26 & -0.51 & 0.11 & 1.46 & 0.03 & 0.19 & -0.20 & 0.12 & 0.20 \\
\hline 4.1 & -0.05 & 0.40 & -0.72 & 0.08 & 2.28 & 0.04 & 0.30 & -0.21 & 0.06 & 0.17 \\
\hline 5.5 & -0.04 & 0.49 & -0.96 & 0.12 & 2.50 & 0.05 & 0.38 & -0.21 & 0.06 & 0.27 \\
\hline
\end{tabular}

\section{CONCLUSION}

A calibration and accuracy study of a pipe laser profiler was carried out, this was performed in relation to systematic errors arising from tolerances from assembly of the optical elements. The specific tolerance that was investigated was the angular misalignment of the conical mirror which projects the laser onto the circumference of the pipework under inspection. A process for aligning the optical elements in translation and orientation was presented which allowed for the angular error to be studied at known points of misalignment. This confirmed the theoretical understanding that the error would manifest itself as a significant translation $\left(15.8 \mathrm{~mm}\right.$ at $5^{\circ}$ within a $120 \mathrm{~mm}$ diameter sample) of the imaged specimen as well as a radius mismeasurement $(1.5 \mathrm{~mm})$. These results highlighted the need for a method to align the optical elements within the profiler to ensure the high levels of accuracy demanded of such systems.

Once aligned using the procedure outlined this error was reduced to $0.12 \mathrm{~mm}$ in positional error and $0.05 \mathrm{~mm}$ in radius error. In practice it is not always possible to manually adjust the optical elements in a laser profiler. Due to this a correction or calibration has been developed by taking advantage of the precisely known location of the profiler within the calibration artefact. By knowing the location of the profiler and making the assumption that the conical mirror is accurately machined, model parameters can be calculated at a finite number of steps around the laser profile image. It is feasible that this process could be carried out as part of the production line with each device paired with a unique calibration data file.

At $4^{\circ}$ misalignment this correction/calibration was shown to correctly image the laser ring within the calibration artefact and when tested on pipe samples with radii ranging from $37.41 \mathrm{~mm}$, to $63.43 \mathrm{~mm}$ reduced the error on average from $2.19 \mathrm{~mm}$ to $0.29 \mathrm{~mm}$. This new software-based calibration approach will be integrated into the deployment system providing accurate dimensioning capability for future industrial internal pipework inspections.

\section{ACKNOWLEDGMENT}

This work was jointly funded by the Research Centre in Non-Destructive Evaluation (RCNDE) and Inspectahire Ltd. The authors would like to thank the additional project partners, National Nuclear Laboratory, Wideblue and Sellafield Ltd.

\section{REFERENCES}

[1] L. J. Bond, P. Ramuhalli, M. S. Tawfik, and N. J. Lybeck, "Prognostics and life beyond 60 years for nuclear power plants," in 2011 IEEE Conference on Prognostics and Health Management, Jun. 2011, pp. 1-7.

[2] T. Vidal-Calleja, J. V. Miró, F. Martín, D. C. Lingnau, and D. E. Russell, "Automatic detection and verification of pipeline construction features with multi-modal data," in 2014 IEEE/RSJ International Conference on Intelligent Robots and Systems, Sep. 2014, pp. 3116-3122.

[3] P. Hansen, H. Alismail, P. Rander, and B. Browning, "Visual mapping for natural gas pipe inspection," The International Journal of Robotics Research, vol. 34, no. 4-5, pp. 532-558, Apr. 2015. [Online]. Available: https://doi.org/10.1177/0278364914550133

[4] A. V. Reyes-Acosta, I. Lopez-Juarez, R. Osorio-Comparan, and G. Lefranc, "3d pipe reconstruction employing video information from mobile robots," Applied Soft Computing, vol. 75, pp. 562-574, Feb. 2019. [Online]. Available: http://www.sciencedirect.com/science/article/pii/ S1568494618306483

[5] L. Jin, N. Miyatsu, E. Kondoh, B. Gelloz, N. Kanazawa, and T. Yoshizawa, "Measurement of diameter of cylindrical openings using a disk beam probe," Optical Review, vol. 25, no. 6, pp. 656-662, Dec. 2018. [Online]. Available: https://doi.org/10.1007/s10043-018-0459-7

[6] N. Stanić, M. Lepot, M. Catieau, J. Langeveld, and F. H. L. R. Clemens, "A technology for sewer pipe inspection (part 1): Design, calibration, corrections and potential application of a laser profiler," Automation in Construction, vol. 75, pp. 91-107, Mar. 2017. [Online]. Available: http://www.sciencedirect.com/science/article/pii/ S0926580516304885

[7] A. D. Tezerjani, M. Mehrandezh, and R. Paranjape, "Pose Estimation of a Dioptric Imaging Sensor with a Circle-Projecting Collimated Laser Moving Inside a Pipeline," in Nonlinear Approaches in Engineering Applications, L. Dai and R. N. Jazar, Eds. Cham: Springer International Publishing, 2015, pp. 281-298. [Online]. Available: http://link.springer.com/10.1007/978-3-319-09462-5

[8] K. Matsui, A. Yamashita, and T. Kaneko, "3-D shape measurement of pipe by range finder constructed with omni-directional laser and omnidirectional camera," in 2010 IEEE International Conference on Robotics and Automation, May 2010, pp. 2537-2542.

[9] P. Buschinelli, T. Pinto, F. Silva, J. Santos, and A. Albertazzi, "Laser Triangulation Profilometer for Inner Surface Inspection of 100 millimeters (4") Nominal Diameter," Journal of Physics: Conference Series, vol. 648, no. 1, p. 012010, 2015. [Online]. Available: http://stacks.iop.org/1742-6596/648/i=1/a=012010

[10] Y. Zhu, Y. Gu, Y. Jin, and C. Zhai, "Flexible calibration method for an inner surface detector based on circle structured light," Applied Optics, vol. 55, no. 5, p. 1034, Feb. 2016. [Online]. Available: https://www.osapublishing.org/abstract.cfm?URI=ao-55-5- 1034

[11] R. He, C. Liu, Z. Zhang, and J. Sun, "Measurement model of the omnidirectional structured-light vision and its calibration method," OSA Continuum, vol. 2, no. 3, pp. 839-847, Mar. 2019. [Online]. Available: https://www.osapublishing.org/osac/abstract.cfm?uri=osac-2- 3-839

[12] R. Summan, G. Dobie, G. West, S. Marshall, C. MacLeod, and S. G. Pierce, "The influence of the spatial distribution of 2-d features on pose estimation for a visual pipe mapping sensor," IEEE Sensors Journal, vol. 17, no. 19, pp. 6312-6321, 2017.

[13] D. Scaramuzza, A. Martinelli, and R. Siegwart, "A Flexible Technique for Accurate Omnidirectional Camera Calibration and Structure from Motion," in Fourth IEEE International Conference on Computer Vision Systems (ICVS'06). New York, NY, USA: IEEE, 2006, pp. 45-45. [Online]. Available: http://ieeexplore.iee.org/document/1578733/

[14] M. Rufli, D. Scaramuzza, and R. Siegwart, "Automatic detection of checkerboards on blurred and distorted images," in 2008 IEEE/RSJ 
International Conference on Intelligent Robots and Systems, Sep. 2008, pp. 3121-3126.

[15] D. Scaramuzza, A. Martinelli, and R. Siegwart, "A Toolbox for Easily Calibrating Omnidirectional Cameras," in 2006 IEEE/RSJ International Conference on Intelligent Robots and Systems, Oct. 2006, pp. 56955701.

[16] S. Urban, J. Leitloff, and S. Hinz, "Improved wide-angle, fisheye and omnidirectional camera calibration," ISPRS Journal of Photogrammetry and Remote Sensing, vol. 108, pp. 72-79, Oct. 2015. [Online]. Available: http://www.sciencedirect.com/science/article/pii/ S0924271615001616

[17] _ "MLPNP - A Real-Time Maximum Likelihood Solution to the perspective-N-Point Problem," ISPRS Annals of Photogrammetry, Remote Sensing and Spatial Information Sciences, vol. III-3, pp. 131-138, Jun. 2016. [Online]. Available: http://www.isprsann-photogramm-remote-sens-spatial-inf- sci.net/III-3/131/2016/isprsannals-III-3-131-2016.pdf

[18] T. Rabbani, "PROTO toolbox." [Online]. Available: http://viztronics.com/tahir/data/PROTO/doc/menu.html

[19] S. Jianbo and C. Tomasi, "Good features to track," in IEEE Computer Society Conference on Computer Vision and Pattern Recognition, 1994, pp. 593-600.

[20] T. J. Atherton and D. J. Kerbyson, "Size invariant circle detection," Image and Vision Computing, vol. 17, no. 11, pp. 795-803, Sep. 1999 [Online]. Available: http://www.sciencedirect.com/science/article/pii/ S0262885698001607

[21] "ATOS Triple Scan | GOM." [Online]. Available: https://www.gom.com/metrology-systems/atos/atos-triple-scan.html

[22] A. Vedaldi and B. Fulkerson, "VLFeat: An open and portable library of computer vision algorithms," http://www.vlfeat.org/, 2008.

[23] C. Mineo, J. Riise, R. Summan, C. N. MacLeod, and S. G. Pierce, "Index-based triangulation method for efficient generation of large three-dimensional ultrasonic C-scans," Insight - Non-Destructive Testing and Condition Monitoring, vol. 60, no. 4, pp. 183-189, Apr. 2018. [Online]. Available: http://www.ingentaconnect.com/content/10.1784/insi.2018.60.4.183

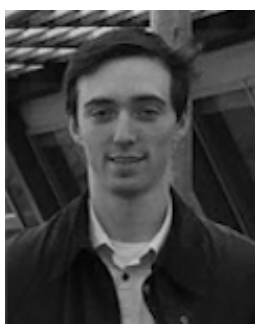

William Jackson received the BEng (Hons) degree from the University of Strathclyde, Glasgow, U.K., in 2015. He is currently an EngD researcher with Inspectahire Ltd./Research Centre for NonDestructive Evaluation based in the Department of Electronic and Electrical Engineering at the University of Strathclyde. His current work focusses on developing automated remote inspection methods for the oil \& gas and nuclear industry.

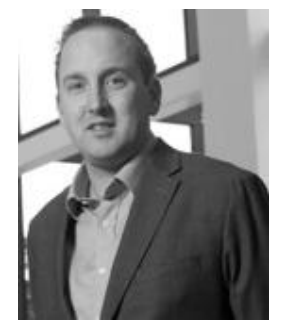

Gordon Dobie is a Senior Lecturer in Electronic \& Electrical Engineering at the University of Strathclyde, UK. He has 12 years' experience and almost 60 publications in automation, structural inspection, sensors and signal processing. Dr Dobie has a strong track record in industrial engagement working with partners in the Oil and Gas, Nuclear and Healthcare sectors to deliver novel solutions to real industrial problems. His work led directly to an onsite robotic inspection of a critical path reprocessing vessel at Sellafield. Dr Dobie has licensed key parts of his team's mobile robotics technology to SME members of the Research Centre for Non-Destructive Evaluation. He currently leads a team of $12 \mathrm{PhD} / \mathrm{EngD}$ students and postdoctoral researchers.

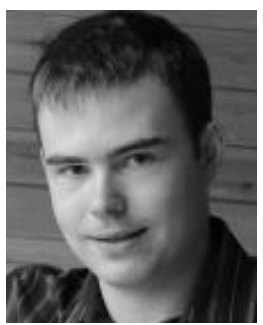

Charles MacLeod is a Lecturer in the Centre for Ultrasonic Engineering. After being awarded a Masters in Electrical and Mechanical Engineering with Distinction at Strathclyde, Charles then went on to undertake a PhD in Automated Non-Destructive Evaluation. While undertaking his PhD Charles, was seconded to Spirit AeroSystems, in Prestwick to undertake Knowledge Exchange activities built on fundamental EPSRC funded research. Charles was awarded the prestigious University of Strathclyde EPSRC Doctoral Prize for 2014, for his work investigating automated NDE. Charles has vast experience in electrical and mechanical engineering areas such as robotics, sensors, electronics, mechanical fixturing and software.

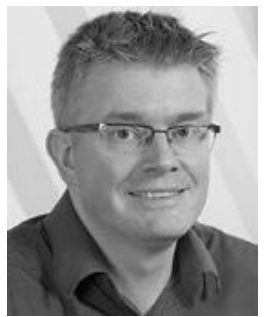

Graeme West received the B.Eng. (Hons.) and $\mathrm{Ph} . \mathrm{D}$. degrees from the University of Strathclyde, Glasgow, U.K., in 1998 and 2002, respectively. He is a Senior Lecturer in the Department of Electronic and Electrical Engineering at the University of Strathclyde. His research interests include intelligent system applications in condition monitoring, diagnostics and prognostics, particularly applied to the nuclear power generation industry.

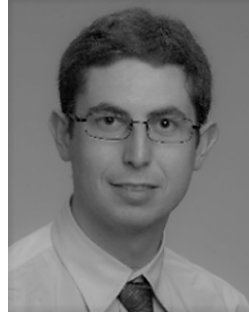

Carmelo Mineo was born in Palermo (Italy) in 1987. He studied Mechanical Engineering at the University of Palermo, where he obtained the Bachelors' degree in 2009 and the Masters' degree in 2011. In 2012, with a background in mechanical engineering, laser ultrasound and NDT testing, he joined the Centre for Ultrasonic Engineering (CUE) of the department of Electrical and Electronic Engineering at the University of Strathclyde to undertake a $\mathrm{PhD}$ in Automated Non-Destructive Inspection of Large and Complex Geometries of Composite Materials. Dr Mineo became a Research Associate of the University of Strathclyde in 2015 and a Research Fellow in 2018. His current research interests comprise all aspects of the operation of robotic cells, including programming, instrument interfacing, data collection and visualization. $\mathrm{He}$ has vast experience in industrial engineering areas such as robotics, sensors, mechanical design, software, system integration, interfacing and control.

Liam McDonald received the MEng degree from the University of Strathclyde, Glasgow, U.K., in 2016. He is currently a PhD researcher in the Department of Electronic and Electrical Engineering at the University of Strathclyde. 